\title{
Human regulatory B cells in health and disease: therapeutic potential
}

\author{
Claudia Mauri and Madhvi Menon
}

Centre for Rheumatology, Division of Medicine, University College London, London, United Kingdom.

\begin{abstract}
Regulatory B cells (Bregs) modulate immune responses predominantly, although not exclusively, via the release of IL-10. The importance of human Bregs in the maintenance of immune homeostasis comes from a variety of immune-related pathologies, such as autoimmune diseases, cancers, and chronic infections that are often associated with abnormalities in Breg numbers or function. A continuous effort toward understanding Breg biology in healthy individuals will provide new opportunities to develop Breg immunotherapy that could prove beneficial in treating various immune-mediated pathologies. In this Review, we discuss findings regarding human Bregs, including their mechanisms of suppression and role in different disease settings. We also propose several therapeutic strategies targeting Bregs for better management of immune disorders.
\end{abstract}

\section{Introduction}

Immune response-associated inflammation is pivotal in protecting the host against foreign pathogens; however, if left unrestrained, it can cause deleterious and often irreversible damage to tissues and organs. Although initiation of inflammatory responses is primarily caused by infection or injury, a variety of autoimmune diseases and cancers can promote chronic inflammation (1). If left unchecked, continuous exposure to proinflammatory soluble mediators, such as TNF- $\alpha$, IL-17, IL-6, and IFN- $\alpha$, contributes to the pathogenesis of several autoimmune and inflammatory disorders, including rheumatoid arthritis (RA), multiple sclerosis (MS), and systemic lupus erythematosus (SLE) (2). To avoid irreversible damage, prompt generation of an antiinflammatory cellular response that minimizes tissue injuries and promotes restoration of homeostasis is required (3). Multiple regulatory pathways that prevent further lymphocytic hyperactivation and restrain existing inflammatory signals are in place to restore immune homeostasis.

In addition to the well-established contribution of Tregs in the maintenance of immune homeostasis, B cells producing IL-10, known as regulatory B cells (Bregs), have been shown to contribute to the maintenance of tolerance (4-6). Immunosuppressive Bregs that express IL-10 and other antiinflammatory mediators are involved in the maintenance of homeostasis in the immune system. The importance of Bregs is emphasized by the different immune-related pathologies that are associated with abnormalities in the number and function of Bregs (4, 6-11). For these reasons, there is increasing interest in better understanding the biology of Bregs and identifying the signals that induce their differentiation in order to exploit their therapeutic potential. This Review summarizes the role of human Bregs in health and different disease settings. We also discuss possible Breg-directed therapeutic strategies that could provide ways of reshaping and resetting the immune system for improved treatment of various diseases.

Conflict of interest: The authors have declared that no conflict of interest exists. Reference information: J Clin Invest. 2017;127(3):772-779.

https://doi.org/10.1172/JCl85113.

\section{Mechanism of suppression}

Bregs are indispensable for the maintenance of tolerance and immune homeostasis, despite representing fewer than $10 \%$ of $\mathrm{B}$ cells in circulation in healthy individuals (4). Breg-mediated suppression occurs primarily via the production of IL-10; therefore, IL-10 is often used as a marker for Breg identification. Detection of IL-10 by intracellular staining makes it difficult to conduct a functional assessment of Bregs; hence other cell surface markers are used to identify "surrogate" Bregs and Breg precursors. Here, we define Bregs as IL-10-producing B cells or B cells that exhibit immune suppression. It is important to note that the identification of Bregs in vitro requires additional stimulation, as IL-10-producing Bregs cannot be identified directly ex vivo. The signals required for Breg differentiation are discussed in the section below.

Multiple subsets of B cells that produce IL-10, often with overlapping surface markers but diverse functions, have been reported and are collectively referred to as Bregs (12). These include IL-10producing $\mathrm{CD} 24^{\mathrm{hi}} \mathrm{CD} 38^{\mathrm{hi}} \mathrm{B}$ cells, CD $24^{\mathrm{hi}} \mathrm{CD} 27^{+} \mathrm{B}$ cells $(\mathrm{B} 10)$, $\mathrm{CD}^{2} 8^{+} \mathrm{CD} 1 \mathrm{~d}^{+} \operatorname{IgM}{ }^{+} \mathrm{CD} 147^{+} \mathrm{GrB}^{+} \mathrm{B}$ cells, CD27 ${ }^{\text {int }} \mathrm{CD} 38^{\text {hi }}$ plasmablasts, and $\mathrm{CD} 19^{+} \mathrm{TIM}^{+} \mathrm{B}$ cells, which have all been shown to suppress proinflammatory responses $(4,13-16)$. Notably, fewer than $20 \%$ of the B cells within these different B cell subsets produce IL-10 and suppress immune responses. IL-10-producing Bregs, most of which express CD24, CD1d, and variable levels of CD27, inhibit the activation of Th1 responses, inhibit the differentiation of Th17 cells, and can also convert $\mathrm{CD} 4^{+} \mathrm{T}$ cells into suppressive Tregs and type 1 regulatory (Tr1) cells $(4,6)$. Although IL-10 is the primary cytokine required for suppression, the engagement of CD80 and CD86 on Bregs enhances the inhibition of Th1 responses $(4,6)$. In addition to $\mathrm{CD}^{+} \mathrm{T}$ cells, IL-10 ${ }^{+}$Bregs inhibit IFN- $\gamma$ production by $\mathrm{CD}^{+} \mathrm{T}$ cells in response to hepatitis $\mathrm{B}$ virus (HBV) infection (17), and suppress TNF- $\alpha$ production following stimulation with LPS and bacterial CpG DNA by activating monocytes (13). More recently, it has been shown that in healthy individuals, Bregs suppress production of the antiviral cytokine IFN- $\alpha$ in plasmacytoid dendritic cells ( $p D C s$ ) via the release of IL-10, suggesting that Bregs are involved in the prevention of collateral damage 
Table 1. Mechanisms of human Breg-mediated suppression

\begin{tabular}{|c|c|c|c|c|}
\hline Subtype & Phenotype & Mechanism of suppression & Target of suppression & References \\
\hline Immature B cells & CD24 $4^{\text {hicD38 }} 8^{\text {hi }}$ & IL-10, PD-L1, CD80, CD86, CD1d & $\mathrm{CD}^{+} \mathrm{T}$ cells, $\mathrm{CD}^{+} \mathrm{T}$ cells, pDCs, iNKT cells & $4,18,21,59$ \\
\hline $\mathrm{CZMB}^{+} \mathrm{B}$ cells & $\mathrm{CD} 8^{+} \mathrm{CD}_{1 \mathrm{~d}^{+}} \operatorname{lgM}^{+} \mathrm{CD} 147^{+}$ & GZMB, IL-10, IDO & $\mathrm{CD4}^{+} \mathrm{T}$ cells & 14 \\
\hline Plasmablasts & $\mathrm{CD} 27^{\text {int }} \mathrm{CD} 38^{\text {hi }}$ & IL-10 & - & 15 \\
\hline- & $\mathrm{CD} 9^{+} \mathrm{CD}_{3}{ }^{+}$ & Adenosine & $\mathrm{CD}^{+} \mathrm{T}$ cells, $\mathrm{CD}^{+} \mathrm{T}$ cells & 22 \\
\hline iBregs & - & TGF- $\beta$, IDO & $\mathrm{CD}^{+} \mathrm{T}$ cells & 20 \\
\hline
\end{tabular}

that is caused by unresolved infections (18). Finally, B regulatory 1 (Br1) cells, identified as $\mathrm{CD} 25^{\mathrm{hi}} \mathrm{CD} 71^{\mathrm{hi}} \mathrm{CD} 73^{\text {lo }} \mathrm{B}$ cells, contribute to the maintenance of allergen tolerance by producing a copious amount of allergen-specific IgG4 antibodies and by suppressing allergen-specific T cell proliferation (19).

Like other immunosuppressive cells, such as Tregs, B cells can differentiate into induced Bregs (iBregs) in response to certain stimuli. It has been previously shown that $\mathrm{T}$ cells expressing cytotoxic T lymphocyte-associated protein 4 (CTLA-4) promote the differentiation of a population of iBregs that modulate immune responses via the production of TGF- $\beta$ and indoleamine 2,3-dioxygenase (IDO), which can convert the interacting T cells into IL-10and TGF- $\beta$-producing Tregs (20). Although the importance of CTLA-4-expressing T cells in the differentiation of Bregs remains to be ascertained, it is possible that this mechanism of expansion exists in vivo as a way to guarantee immunosuppression in the presence of excessive inflammation. Furthermore, CD1d-mediated lipid antigen presentation by IL-10-producing $\mathrm{CD} 24^{\text {hi }} \mathrm{CD} 38^{\text {hi }} \mathrm{B}$ cells (CD24 ${ }^{\text {hi }} \mathrm{CD} 38^{\text {hi }}$ Bregs) is important for maintaining the number and function of induced NKT cells (iNKT cells), a cell subset with immunosuppressive capacity (21).

$\mathrm{CD} 39^{+} \mathrm{CD}_{3}{ }^{+} \mathrm{B}$ cells drive a shift from an ATP-driven proinflammatory environment to an adenosine-induced antiinflammatory milieu (22). CD39 and CD73 are ectonucleotidases that can hydrolyze exogenous adenosine triphosphate (ATP) to adenosine $5^{\prime}$-monophosphate (5'-AMP) and adenosine. It has been suggested that upon in vitro activation with CD4OL and IL-4,
$\mathrm{CD} 9^{+} \mathrm{CD}^{2} 3^{+}$Bregs inhibit $\mathrm{CD} 4^{+}$and $\mathrm{CD}^{+} \mathrm{T}$ cell proliferation via the production of $5^{\prime}$-AMP (22). It is noteworthy that although not yet described in human studies, IL-35 has been shown to be important in Breg-mediated suppression. In a mouse model of MS, experimental autoimmune encephalomyelitis (EAE), mice lacking B cell-specific IL-35 production developed exacerbated disease and also displayed improved resistance to Salmonella infection (23). The growing number of suppressive mechanisms ascribed to Bregs suggests that Bregs play a multifaceted role in immune regulation. The diverse mechanisms of immune suppression by Bregs are summarized in Table 1.

\section{Signals required for Breg differentiation}

In order to be activated and exhibit suppressive functions, Bregs require the engagement of combinations of several molecules, including TLR, CD40, and/or B cell receptor (BCR), as well as CD80, CD86, and cytokine receptors. While the majority of evidence demonstrating the relevance of costimulatory signals, TLRs, and cytokines comes from murine studies, several of these signals have also been found necessary for the differentiation of Bregs in humans. It is noteworthy that to detect a distinct population of IL-10-expressing B cells in vitro using flow cytometry, an additional robust stimulation with phorbal 12-myristate 13-acetate, ionomycin, and brefeldin $\mathrm{A}$ is used, either alone or in combination with other stimuli. Signals controlling the activation of Bregs have been discussed in detail elsewhere (24) and are summarized here in Figure 1.
Figure 1. Induction of Bregs. IL-10-producing Bregs that express regulatory genes are generated as a consequence of immune activation. The signals that induce the differentiation of Bregs include inflammatory stimuli (via TLRs), costimulatory signals (CD40, CD80, CD86), microbiota, and cytokines (IFN- $\alpha / \beta$, IL-1 $\beta$, IL-6, IL-21, and BAFF).

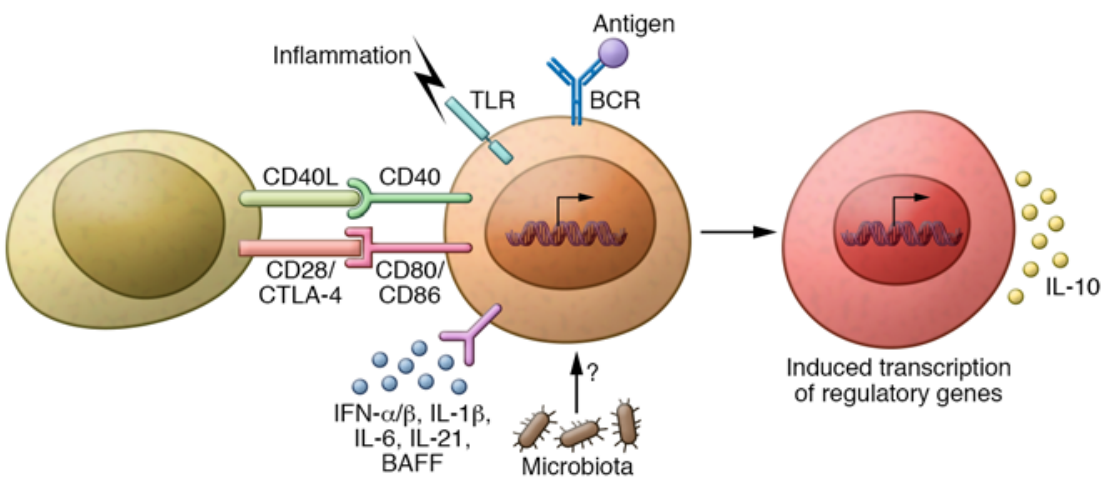




\section{Role in disease}

Autoimmunity. Numerical and functional Breg defects have been described in several autoimmune diseases, including SLE, RA, MS, and psoriasis $(4,6,21,25,26)$. Multiple studies have identified inverse correlations between Breg numbers and function and disease activity. In the context of autoimmunity, this raises two possible scenarios. First, the loss of immune suppression driven by Bregs contributes to inflammation. The second scenario is that the reduced Breg numbers and function are a consequence of chronic inflammation. Because of the current limitations of human Breg studies, evidence supporting either scenario needs to be extrapolated entirely from murine studies. Chimeric mice with IL-10 deficiency specific to B cells have been shown to develop exacerbated arthritis as well as EAE due to increases in Th1 and/or Th17 responses compared with WT mice $(27,28)$. Furthermore, adoptive transfer of mouse Breg subsets has been shown to suppress various autoimmune diseases, including arthritis, EAE, and lupus $(5,29-31)$. It is noteworthy that inflammatory signals have been reported to expand functional Bregs, rather than reduce numbers $(32,33)$. Novel therapeutics specifically targeting Bregs will help address these remaining questions in the future.

Multiple sclerosis. Suppressive B cells were first identified in patients with MS: MS patients infected with helminths displayed an increased frequency of IL-10-producing $\mathrm{CD} 19^{+} \mathrm{CD} 1 \mathrm{~d}^{\text {hi }} \mathrm{B}$ cells as well as a better clinical outcome. The B cells isolated from MS patients with helminth infection suppressed proliferation and IFN- $\gamma$ production by T cells (34). Although in human studies, it is difficult to conclusively establish the exact mechanism of action, the amelioration of disease symptoms in helminthinfected MS patients was speculated to be due to the expansion of IL-10-producing B cells. More recently, in relapsing-remitting MS (RRMS), a reduction in IL-10-producing B cells was found in patients experiencing relapse compared with patients in remission and compared with healthy individuals (25). Interestingly, IFN- $\beta$ therapy, a treatment for RRMS, has been shown to work via the expansion of CD24 ${ }^{\text {hi }} \mathrm{CD} 38^{\text {hi }}$ Bregs in patients (35). In the same study, the authors took advantage of an experimental model of MS to show that while WT mice respond to IFN- $\beta$ therapy, B celldeficient mice were refractory to the same therapeutic regime, confirming that $\mathrm{B}$ cells mediate the antiinflammatory effects of IFN- $\beta$ therapy (35). Similarly, other studies have reported an expansion of Breg phenotype and function upon treatment of MS patients with fingolimod (a sphingosine-1-phosphate modulator) and alemtuzumab (an anti-CD52 therapy) (36-38). Furthermore, rituximab therapy in MS patients has been shown to improve clinical response by ablating pathogenic IL-6-producing B cells (39). Taken together, immunomodulatory treatments in MS patients might work, in part, by inducing a shift in B cells toward a more antiinflammatory phenotype.

Systemic lupus erythematosus. Patients with active SLE are characterized by a numerical and functional deficiency in circulating Bregs. Several studies have now shown that the defect is associated with the inability of immature $\left(\mathrm{CD} 19^{+} \mathrm{CD} 24^{\mathrm{hi}} \mathrm{CD} 38^{\mathrm{hi}}\right)$ $\mathrm{B}$ cells to differentiate into Bregs in response to signals known to be pivotal for their differentiation $(4,18,40,41)$. Whereas healthy immature B cells respond to CD40 stimulation and differentiate into Bregs, CD2 $4^{\text {hi }} \mathrm{CD} 38^{\text {hi }} \mathrm{B}$ cells isolated from SLE patients dis- play impaired IL-10-producing capacity upon CD40 activation, and are unable to suppress Th1 responses $(4,40)$. Independently, another study demonstrated that large $\mathrm{CD} 19^{+} \mathrm{FSC}^{\text {hi }}$ polyclonally activated B cells (iBregs) from SLE patients display a significantly reduced ability to suppress Th cell responses compared with B cells from healthy individuals (41). Additionally, stimulation with TLR9-activated pDCs induces a multifold expansion of immunosuppressive IL-10-producing $\mathrm{CD} 24^{+} \mathrm{CD} 38^{\text {hi }}$ Bregs in healthy individuals but not SLE patients (18).

Our group has recently identified the reason for the loss of Breg function in patients with SLE, an autoimmune disease characterized by increased IFN- $\alpha$ levels and an IFN- $\alpha$-induced gene signature (18). Our results indicate that the level of exposure to the proinflammatory cytokine IFN- $\alpha$ is important in determining immature B cell fate: whereas low concentration of IFN- $\alpha$ simultaneously expands both Bregs and plasmablasts, high concentration of IFN- $\alpha$ skews B cell differentiation in favor of plasmablasts but fails to expand Bregs (18). In these patients, increased IFN- $\alpha$ signaling is mirrored by a loss of functional Bregs as well as an increase in autoantibody-producing plasma cells that contribute to disease pathogenesis (18). Further analysis revealed that the Breg defects were also associated with alterations in phosphorylation of signals downstream of the IFN- $\alpha / \beta$ receptor, namely STAT1 and STAT3 $(18,42)$. This is most likely due to chronic exposure to IFN- $\alpha$ in vivo. In addition, functionally impaired $\mathrm{CD} 24^{\text {hi }} \mathrm{CD} 38^{\text {hi }}$ Bregs isolated from patients with SLE fail to restrain IFN- $\alpha$ production by hyperactivated pDCs (18). This suggests that the immune-regulatory pDC-Breg feedback loop that is in place in healthy individuals is dysfunctional in SLE patients.

Because of the multiple abnormalities in the B cell compartment in SLE and their hypothesized role in SLE pathogenesis, patients are often treated with rituximab (B cell depletion) therapy $(43,44)$. Upon B cell repopulation, a higher immature-to-memory ratio has been associated with long-term remission $(45,46)$, suggesting that repopulation with $\mathrm{CD} 24{ }^{\text {hi }} \mathrm{CD} 38^{\text {hi }}$ Bregs might be associated with improved clinical response. This concept is supported by independent studies evaluating SLE patients treated with rituximab in which the repopulation of $\mathrm{CD} 24^{\mathrm{hi}} \mathrm{CD} 38^{\text {hi }}$ Bregs with restored suppressive functions corresponded with an improved clinical response $(18,21)$. Patients responding to rituximab therapy display normal STAT1 and STAT3 activation and have restored IL-10 production by repopulated B cells (18). Furthermore, the restored $\mathrm{CD} 24^{\mathrm{hi}} \mathrm{CD} 38^{\mathrm{hi}}$ Breg function corresponded to normalized activation of pDCs that were otherwise hyperactivated in SLE patients (18). Another study evaluating the iNKT cell response in rituximab-treated SLE patients provides further evidence for the role of Bregs in the improved clinical response. iNKT cells are scarcely present in patients with SLE, and we and others have shown that this is due to dysfunctional interactions with aberrant B cells (21, 47). However, following B cell repopulation, normalization of CD1d expression on newly repopulated $\mathrm{CD} 19^{+} \mathrm{CD} 24^{\text {hi }} \mathrm{CD} 38^{\text {hi }} \mathrm{B}$ cells corresponded with normalization of the iNKT cell number and function (21), suggesting that Bregs are important in the maintenance of homeostatic levels of iNKT cells. Taken together, these in vitro studies suggest that newly repopulated Bregs in SLE patients might suppress inflammation by empowering several other cells of the immune system with immunosuppressive functions. 
Rheumatoid arthritis. In patients with RA, CD24 $4^{\text {hi }} \mathrm{CD} 38^{\text {hi }}$ Bregs are numerically impaired in comparison with healthy individuals and fail to suppress Th17 responses and convert CD4 ${ }^{+} \mathrm{T}$ cells into Tregs (6). The frequency of Bregs in RA patients was shown to negatively correlate with disease activity. In agreement with this finding, three recent studies have reported reduced frequencies of B10 cells, IL $-10^{+} \mathrm{TIM}^{+} \mathrm{B}$ cells, and $\mathrm{IL}-10^{+} \mathrm{CD}^{+} \mathrm{CD} 1 \mathrm{~d}^{\text {hi }} \mathrm{B}$ cells in RA patients compared with healthy controls (48-50). In contrast, one study reported an increase in $\mathrm{IL}-10^{+}$Bregs in RA patients compared with healthy controls (51). The disparity between the studies is likely due to the differences in stimuli used for inducing IL-10 production by $B$ cells in vitro. Whereas the studies showing a decrease in IL-10 Bregs used either TLR or CD40 activation of B cells, the study reporting an increase in Bregs used CD40 ligation in combination with TLR activation. It is possible that using this combination of stimuli may overcome the defects shown in the previous studies.

Other autoimmune diseases. Studies of patients with other autoimmune diseases are extremely limited in comparison with SLE, MS, or RA.

In patients with pemphigus, an organ-specific autoimmune bullous disease $(52), C D 24{ }^{\text {hi }} C D 38^{\text {hi }} B$ cells displayed reduced IL-10 production upon long-term stimulation and a significantly decreased ability to suppress Th1 responses (10). Interestingly, rituximab-treated pemphigus patients who responded to therapy displayed increased frequencies of $\mathrm{CD} 24^{\mathrm{hi}} \mathrm{CD} 38^{\mathrm{hi}}$ $B$ cells and IL-10 production compared with untreated patients or patients not responding to therapy (53). It is possible that Bregs may contribute to restoration of tolerance in rituximabresponsive pemphigus patients.

Inflammatory bowel disease is a chronic inflammatory disease that has two major clinically defined forms: Crohn's disease and ulcerative colitis (54). In patients with both Crohn's disease and ulcerative colitis compared with healthy controls, IL-10-producing Bregs are significantly reduced in frequency (11). Similar findings have been reported in patients with type 1 diabetes (55), psoriasis $(26)$, and systemic sclerosis $(56,57)$, in which decreases in suppressive IL-10-producing Bregs have been associated with progression of disease. While further studies are required to understand the mechanisms of Breg-mediated suppression in these diseases, there is sufficient evidence to conclude that Bregs are numerically deficient in autoimmune diseases and possibly contribute to the loss of immune tolerance.

Infection. The role of Bregs in bacterial and parasitic infections has been discussed in detail elsewhere (58). Here, we focus on the role of human Bregs in the immune response during viral infections, such as HIV and HBV infection. In patients infected with HIV, an increase in IL-10-producing CD $24^{\text {hi }} \mathrm{CD} 38^{\text {hi }}$ Bregs has been shown to correlate positively with the viral load (59). In the same paper, Bregs were shown to suppress HIV-1-specific CD8 ${ }^{+} \mathrm{T}$ cell responses in an IL-10- and PD-L1-dependent manner (59). The suppressive role of Bregs during HIV infection was confirmed by depletion of Bregs from peripheral blood mononuclear cells (PBMCs) in vitro, which resulted in restored $\mathrm{CD} 8^{+} \mathrm{T}$ cell effector function as well as clearance of infected CD $4^{+}$T cells (59). Similarly, the frequency of another subset of IL-10-producing Bregs with a CD19+ ${ }^{+}$IM $^{+}$phenotype has been shown to correlate with viral load and effectively inhibit HIV-specific T cell responses in an IL-10-dependent manner
(16). A more recent study showed that Bregs inhibit antigen presentation and $\mathrm{CD}^{+} \mathrm{T}$ cell proliferation and impair anti-HIV cytotoxic $\mathrm{T}$ lymphocyte functions in HIV-infected patients via the release of IL-10 and PD-1/PD-L1 interaction (60). Similarly, in patients with chronic HBV, there was an expansion of IL-10-producing CD24 $4^{\text {hi }}$ $\mathrm{CD} 38^{\text {hi }} \mathrm{B}$ cells, and their frequency correlated with hepatic flares. $\mathrm{CD} 24^{\text {hi }} \mathrm{CD} 38^{\text {hi }}$ Bregs were found to suppress HBV-specific CD8 ${ }^{+}$ T cell responses in an IL-10-dependent manner (17). CD24 ${ }^{\text {hi }} \mathrm{CD} 38^{\text {hi }}$ Bregs in chronic HBV patients suppress Th1 and Th17 responses as well as convert $\mathrm{CD} 4^{+} \mathrm{T}$ cells into Tregs (61). Taken together, these studies implicate a role for $\mathrm{CD} 24^{\mathrm{hi}} \mathrm{CD} 38^{\mathrm{hi}}$ Bregs in hindering viral eradication during an infection.

Allergy. Bregs have been shown to play a role in allergen tolerance and contribute to the suppression of various allergic diseases. In patients with allergic asthma, a reduced expansion of IL-10producing $\mathrm{CD} 24^{\mathrm{hi}} \mathrm{CD} 27^{+}$Bregs was reported in response to LPS stimulation (8). Moreover, LPS-stimulated B cells from patients induced a weaker IL-10 response by dust mite allergen-activated $\mathrm{T}$ cells. A similar reduction in the frequency of IL-10-producing $\mathrm{CD} 24^{\text {hi }} \mathrm{CD} 27^{+}$Bregs was also reported in patients with allergic rhinitis (9). Other studies evaluating Bregs in patients with milk allergy have demonstrated a decrease in IL-10-producing $\mathrm{CD}^{+} \mathrm{B}$ cells upon in vitro restimulation with casein. In contrast, this population of Bregs remained unchanged or increased in the milk-tolerant group $(62,63)$. Similar observations were observed in beekeepers who exhibited tolerance to the bee venom allergen phospholipase $\mathrm{A}_{2}\left(\mathrm{PLA}_{2}\right)$. PLA $\mathrm{A}_{2}$-specific Br1 cells produced allergen-specific IgG4 antibodies and suppressed allergen-specific $T$ cell responses in an IL-10-dependent manner (19).

Cancer. Studies from mouse models have provided extensive evidence supporting an important role for Bregs in tumor immunology. In a mouse model of breast cancer, a subset of CD25 2 hi $69^{\text {hi }}$ tumor-evoked Bregs was reported to facilitate lung metastasis by inducing the differentiation of $\mathrm{FoxP}^{+}$Tregs in a TGF- $\beta$-dependent manner (64). Interestingly, tumor-evoked Bregs expressed high levels of CD80 and CD86, suggesting that CD80- and CD86-mediated contact between Bregs and their target cells is important both in suppression of effector $\mathrm{T}$ cell response and in the differentiation of Breg-induced Tregs. More recently, it has been suggested that tumor-infiltrated B cells develop immune-suppressive properties via enhanced expression of TGF- $\beta$, PD-L1, CD86, and IL-10 (65). In this model, tumor-infiltrating B cells that were not initially intrinsically suppressive developed a Breg phenotype upon exposure to the tumor microenvironment (65). Some of the identified factors promoting a Breg phenotype are tumor-derived metabolites of 5-lipoxygenase in a breast cancer model, and placental growth factor (PIGF) in gliomas $(66,67)$. The role of Bregs in promoting tumor progression is supported by the finding that IL-10-producing Bregs inhibit lymphoma depletion during anti-CD20 therapy in mice (68). Thus far, there are very few reports on the role of Bregs in human cancer studies. Granzyme B-expressing (GZMBexpressing) human B cells with a $\mathrm{CD} 38^{+} \mathrm{CD} 1 \mathrm{~d}^{+} \operatorname{IgM} \mathrm{M}^{+} \mathrm{CD} 147^{+}$phenotype have been shown to infiltrate tumors and inhibit $\mathrm{CD} 4^{+} \mathrm{T}$ cell responses (14). GZMB Bregs were induced by IL-21-producing $\mathrm{T}$ cells and also expressed IL-10, IDO, and CD25 (14). Both GZMB ${ }^{+}$ $\mathrm{B}$ cells and IL-21+ $\mathrm{T}$ cells were identified within the microenvironment of various tumor types, including breast, cervical, and ovar- 


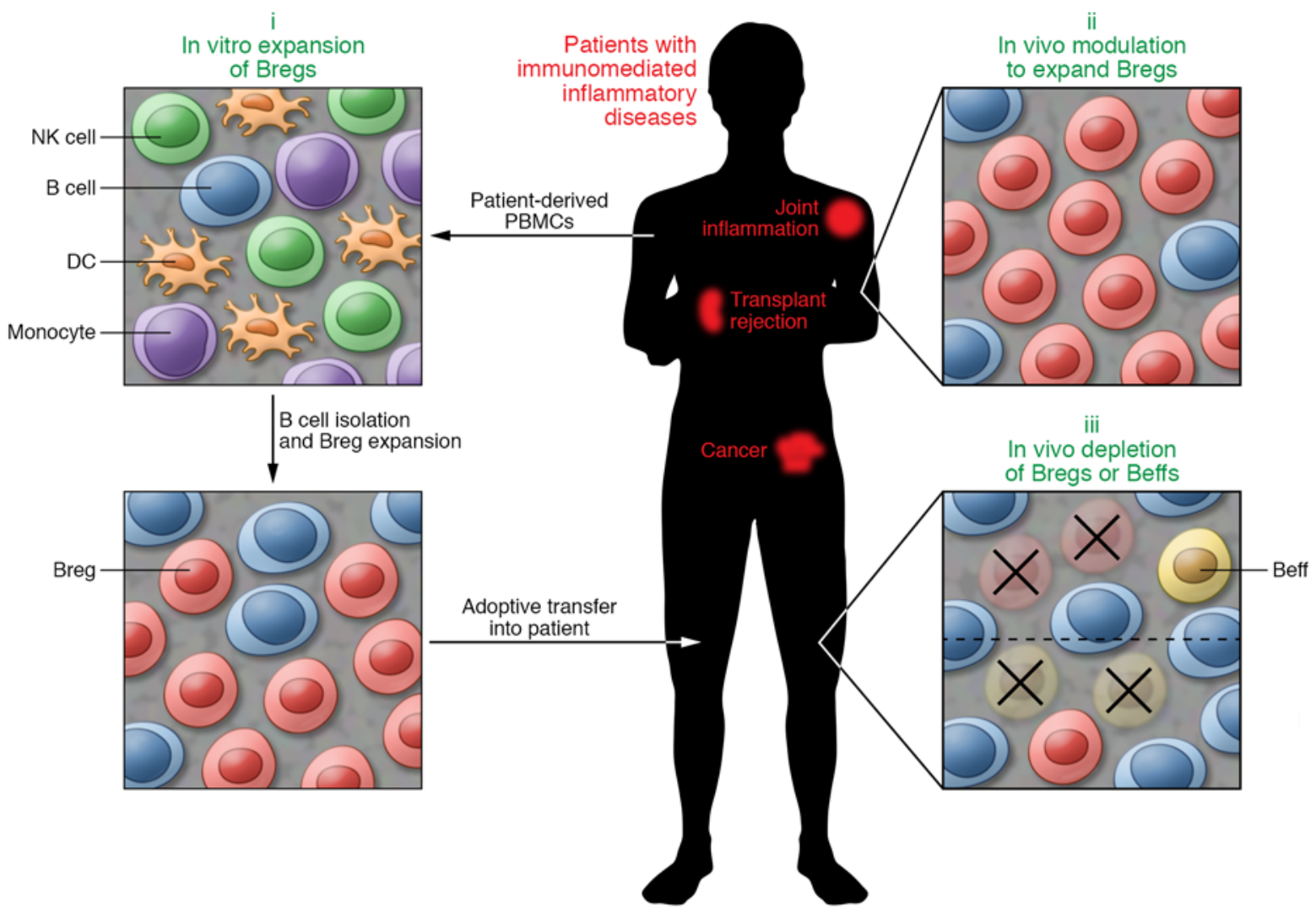

Figure 2. Potential Breg-targeted therapies. Therapeutic interventions targeting Bregs could provide improved approaches for the treatment of immune-mediated diseases. (i) Ex vivo expansion of Bregs. Stimulation of B cells isolated from patient-derived PBMCs to expand Bregs, followed by adoptive transfer of FACS-sorted Bregs, could suppress inflammation and reestablish tolerance. (ii) In vivo modulation to expand Bregs. The recent identification of stimuli that induce Breg differentiation provides new opportunities to induce a shift in B cells toward a more regulatory or antiinflammatory phenotype. (iii) Depletion of Bregs or Beffs. Targeted depletion of specific B cell subsets would provide more advantages over currently used total B cell depletion therapies in the treatment of cancers and other immune disorders.

ian carcinomas (14). These findings are particularly significant, as they confirm the existence of B cells with a regulatory phenotype in solid tumor infiltrates, where they would suppress antitumor immune responses. Improved understanding of Breg induction and function in tumor immunology could result in Breg-targeted therapies that enhance antitumor immunity.

Transplantation. CD $24^{\mathrm{hi}} \mathrm{CD} 38^{\mathrm{hi}}$ Bregs appear to play an important role in establishing transplant tolerance by suppressing effector $\mathrm{T}$ cell responses (69). In studies of allogeneic hematopoietic stem cell transplant recipients, Bregs from patients with chronic graftversus-host disease (cGVHD) displayed reduced IL-10-producing capacity compared with patients without cGVHD and healthy donors (7). Furthermore, renal transplant recipients with stable graft function have been reported to display significantly higher levels of IL-10-producing CD2 ${ }^{\text {hi }} \mathrm{CD} 38^{\text {hi }}$ Bregs than immunosuppressed patients, and these levels were comparable to healthy controls (70). More recently, the ratio of IL-10/TNF- $\alpha$ expression by $\mathrm{CD} 24^{\text {hi }} \mathrm{CD} 38^{\text {hi }} \mathrm{B}$ cells, as a measure of the cytokine polarization of Bregs, has been shown to be a better indicator of Breg function than IL-10 expression alone (71). Whereas there was no difference in $\mathrm{CD} 24^{\mathrm{hi}} \mathrm{CD} 38^{\mathrm{hi}} \mathrm{B}$ cell IL-10 expression between renal transplant patients with stable graft function and those patients showing graft rejection, the IL-10/TNF- $\alpha$ ratio was lower in those patients who subsequently underwent rejection events (71). Further evidence of the importance of Bregs in transplant tolerance has been shown in a recent prospective study in renal transplant patients, where increased $\mathrm{CD} 24^{\mathrm{hi}} \mathrm{CD} 38^{\mathrm{hi}} \mathrm{B}$ cell numbers were found to be associated with reduced rejection rates (72). Interestingly, cord blood (CB) has been identified as a rich source of immunosuppressive Bregs (73). CB-derived Bregs have been shown to protect CB transplant recipients from $c G V H D$ disease. Altogether, these studies support a role for Bregs as a biomarker for transplantation tolerance and shed some light on its therapeutic potential.

\section{Therapeutic potential of Bregs}

The majority of current treatments for various immune-related pathologies target the symptoms of the conditions rather than offer cures. Moreover, most treatments remain toxic and ineffective when given to the patient over a long period of time. In the context of autoimmunity, long-term use of steroids and immunosuppressive drugs increases the risk of life-threatening infections. For these reasons, cellular immunotherapy involving highly targeted removal or modification of only those immune cells that drive disease progression is becoming increasingly popular. This approach has already proven highly effective in the treatment of various cancers, with its low toxicity and increased ability to eradicate tumors (74). 
As detailed above, Bregs are important modulators of the immune response and promote immunological tolerance. Whereas certain conditions such as autoimmune diseases and transplantation require an expansion of immunosuppressive Bregs, other diseases such as cancers and chronic infections may benefit from Breg depletion (75). Thus, strategies designed to isolate, expand, infuse, or deplete Bregs would provide a new window of opportunity to treat various immune-mediated disorders (summarized in Figure 2).

Depletion of Bregs. The use of B cell depletion therapy (i.e., rituximab) has shown some success in the treatment of autoimmune diseases $(43,44)$. However, elimination of all B cells to treat autoimmunity is disadvantageous, as it results in the depletion of Bregs that suppress inflammation. It would therefore be advantageous to be able to selectively deplete Bregs or effector B cell subsets depending on the disease context. One of the major limiting factors to using this approach is the lack of surface markers specific for Bregs. Although several markers, alone or in combination, have been shown to identify the majority of IL-10-producing Bregs, they are not sufficiently Breg-specific to use in cellular therapy $(4,13$, $15,76)$. Identification of Breg-specific markers could result in the development of depletion therapies specifically targeting Bregs or effector B cells (Beffs). For example, enhanced expression of PD-L1 by tumor-infiltrated B cells (a trait particularly attributed to Bregs) provided a rationale for PD-1 blockade in the treatment of malignant B cell lymphomas (77). PD-1 blockade has been reported as highly effective in the treatment of refractory Hodgkin's disease and partially effective in the treatment of patients with relapsed diffuse large B cell lymphoma $(78,79)$. Collectively, this suggests that new therapies targeting Breg activity could be a promising approach to treat certain cancers and chronic infections.

In vivo manipulation or ex vivo expansion of Bregs. Recent emerging evidence suggests that the environmental milieu in which B cells differentiate plays a pivotal role in the induction of Bregs. In addition to CD40, TLR, and BCR signaling, which are known to be important for Breg activation and function, there is emerging evidence suggesting that inflammatory cytokines play a critical role in the induction of immunosuppressive Bregs. For example, an expansion of Bregs is observed upon exposure to inflammatory cytokines, such as IL-1 $\beta$, IL-6, IL-21, IFN- $\beta$, IFN- $\alpha$, and B cell activating factor (BAFF) $(18,32,33,35,80,81)$. This effect is further enhanced by coactivation via TLRs and/or CD40 $(18,82,83)$. While these studies support the possibility of in vivo expansion of Bregs, stimuli such as inflammatory cytokines carry the potential risk of triggering undesirable proinflammatory responses from various cell types. The possibility of serious adverse effects that can arise from systemic administration of these cytokines must be taken into consideration. Indeed, the expansion of Bregs by proinflammatory cytokines appears to be tightly regulated by the strengths of the signals that they receive; using the wrong concentration of the proinflammatory cytokine may lead to effector rather than regulatory B cell expansion. In patients with autoimmunity, chronic exposure of B cells to elevated levels of proinflammatory cytokines results in a reduction in functional Bregs that are unable to restore tolerance (18). Importantly, antiinflammatory cytokines may also be important in the differentiation of Bregs. In a mouse model of experimental autoimmune uveitis, the antiinflamma- tory cytokine IL-35 has been shown to induce a population of Bregs that suppress development and progression of disease (84). Additionally, commensal bacteria have been reported to play a role in Breg expansion. In arthritic mice, gut microbiota-induced IL- $1 \beta$ and IL- 6 production directly promoted Breg differentiation and IL-10 production (32). The importance of microbiota in the expansion of Bregs was confirmed in mice treated with antibiotics that displayed a decrease in Breg numbers compared with untreated mice. Thus, better understanding of the signals that drive Breg expansion could provide new and improved strategies for the in vivo expansion of Bregs.

\section{Challenges and outstanding questions}

Therapies targeted to modify Bregs show great potential in the treatment of autoimmune diseases, cancers, and chronic infections. However, there are several issues that must be addressed in order to develop Breg-targeted therapies:

Plasticity and stability of Bregs. How do we ensure that adoptively transferred Bregs maintain their phenotype and function in vivo? Breg subsets have been identified at different stages of $B$ cell development, from an early immature stage to a late plasma cell stage $(4,15,84)$. These multiple Breg subsets could represent a unique functional Breg lineage that changes phenotype in response to the microenvironmental input. It is unknown whether Bregs develop into Beffs under chronic inflammatory conditions. At present, only one study, using mouse models, reports that Bregs differentiate into antibody-secreting cells after transient IL-10 production in vivo (85). Further investigations on the plasticity and functional stability of Bregs are necessary to understand how to maintain a prolonged Breg phenotype.

Stimuli to expand Bregs. Activation via BCR, TLR, or CD40, as well as cytokines, has been shown to activate and expand Bregs. Furthermore, different combinations of stimuli have been used to quantify Bregs in various diseases. It would be important to compare the different stimuli used in order to identify stimuli that uniquely expand Bregs but not proinflammatory responses from other cell types. We also need to understand whether modulating signals in vivo can provide a long-term favorable environment for Breg differentiation.

Efficient transfer of Bregs. Another important question is what quantity of Bregs to transfer for effective therapy; while too few Bregs may be insufficient to suppress inflammation, too many Bregs could result in increased immune suppression-related disorders such as cancer and risk of infection flares. Moreover, how can we ensure that the transferred Bregs will travel to the target site and suppress disease? To treat diseases such as RA, it is crucial that the Bregs migrate to the inflamed joints. While there is some evidence from murine studies showing that Bregs migrate to sites of inflammation (65), this requires further evaluation. It must be noted that while in vitro culture studies provide an indication of Breg function in vivo, they are not truly representative of Bregs at the site of inflammation. For instance, the stimuli used to expand and identify Bregs in vitro might be different from stimuli in the surrounding tissue microenvironment. Further efforts to characterize Bregs directly from patient material, particularly at the site of inflammation, are vital in understanding the role of Bregs in different disease states. 
Efficacy of Breg therapies compared with immunosuppressive therapies. Finally, would Breg therapy provide a more effective treatment than current immunosuppressive regimens? The improved management of various diseases using current treatments would require Breg-targeted treatment to provide long-term cure with minimal side effects. Future research efforts to address these questions will pave the way for novel Breg-based cellular therapies.

\section{Conclusion}

Over the past decade a wealth of studies have demonstrated that Bregs are crucial in the maintenance of immune tolerance and in the suppression of inflammation. We have discussed our understanding of human Bregs and proposed several therapeutic strategies targeting Bregs for improved management of immune-mediated disorders. Despite the presently unanswered questions and theoret- ical risks, the future of Breg-targeted therapies shows great promise and could provide a more improved approach to treat various immune-related pathologies. Further investigation into developing Breg-based immunotherapies could enable their application for the treatment of immune disorders in the forthcoming decade.

\section{Acknowledgments}

We thank P.A. Blair, E.C. Rosser, and S. Thomson for their comments on the manuscript. M. Menon is funded by a European Commission FP7 program grant (HEALTH-F2-2013-602114).

Address correspondence to: Claudia Mauri, Centre for Rheumatology, Division of Medicine, University College London, 5 University Street, London WC1E 6JF, United Kingdom. Phone: 44.0.2031082155; E-mail: c.mauri@ucl.ac.uk.
1. Medzhitov R. Inflammation 2010: new adventures of an old flame. Cell. 2010;140(6):771-776.

2. Moudgil KD, Choubey D. Cytokines in autoimmunity: role in induction, regulation, and treatment. J Interferon Cytokine Res. 2011;31(10):695-703.

3. Chaudhry A, Rudensky AY. Control of inflammation by integration of environmental cues by regulatory T cells. J Clin Invest. 2013;123(3):939-944.

4. Blair PA, et al. CD19(+)CD24(hi)CD38(hi) B cells exhibit regulatory capacity in healthy individuals but are functionally impaired in systemic Lupus Erythematosus patients. Immunity. 2010;32(1):129-140.

5. Carter NA, et al. Mice lacking endogenous IL-10-producing regulatory B cells develop exacerbated disease and present with an increased frequency of Th1/Th17 but a decrease in regulatory T cells. J Immunol. 2011;186(10):5569-5579.

6. Flores-Borja F, et al. CD $19^{+} \mathrm{CD} 24^{\text {hi }} \mathrm{CD} 38^{\text {hi }} \mathrm{B}$ cells maintain regulatory $\mathrm{T}$ cells while limiting TH1 and TH17 differentiation. Sci Transl Med. 2013;5(173):173ra23.

7. Khoder A, et al. Regulatory B cells are enriched within the IgM memory and transitional subsets in healthy donors but are deficient in chronic GVHD. Blood. 2014;124(13):2034-2045.

8. van der Vlugt LE, et al. CD24(hi)CD27(+) B cells from patients with allergic asthma have impaired regulatory activity in response to lipopolysaccharide. Clin Exp Allergy. 2014;44(4):517-528.

9. Kamekura R, et al. Alteration of circulating type 2 follicular helper T cells and regulatory B cells underlies the comorbid association of allergic rhinitis with bronchial asthma. Clin Immunol. 2015;158(2):204-211.

10. Zhu HQ, et al. Impaired function of CD19(+) CD24(hi) CD38(hi) regulatory B cells in patients with pemphigus. Br J Dermatol. 2015;172(1):101-110.

11. Oka A, et al. Role of regulatory B cells in chronic intestinal inflammation: association with pathogenesis of Crohn's disease. Inflamm Bowel Dis. 2014;20(2):315-328.

12. Mauri C, Menon M. The expanding family of regulatory B cells. Int Immunol. 2015;27(10):479-486.

13. Iwata $\mathrm{Y}$, et al. Characterization of a rare IL-10-competent B-cell subset in humans that parallels mouse regulatory B10 cells. Blood. 2011;117(2):530-541.
14. Lindner S, et al. Interleukin 21-induced granzyme B-expressing B cells infiltrate tumors and regulate T cells. Cancer Res. 2013;73(8):2468-2479.

15. Matsumoto M, et al. Interleukin-10-producing plasmablasts exert regulatory function in autoimmune inflammation. Immunity. 2014;41(6):1040-1051.

16. Liu J, et al. IL-10-producing B cells are induced early in HIV-1 infection and suppress HIV-1-specific T cell responses. PLoS One. 2014;9(2):e89236.

17. Das A, et al. IL-10-producing regulatory B cells in the pathogenesis of chronic hepatitis B virus infection. J Immunol. 2012;189(8):3925-3935.

18. Menon M, Blair PA, Isenberg DA, Mauri C. A regulatory feedback between plasmacytoid dendritic cells and regulatory B cells is aberrant in systemic lupus erythematosus. Immunity. 2016;44(3):683-697.

19. van de Veen $W$, et al. IgG4 production is confined to human IL-10-producing regulatory B cells that suppress antigen-specific immune responses. J Allergy Clin Immunol. 2013;131(4):1204-1212.

20. Nouël A, et al. B-Cells induce regulatory T cells through TGF- $\beta$ /IDO production in A CTLA-4 dependent manner. JAutoimmun. 2015;59:53-60.

21. Bosma A, Abdel-Gadir A, Isenberg DA, Jury EC, Mauri C. Lipid-antigen presentation by CD1d(+) B cells is essential for the maintenance of invariant natural killer T cells. Immunity. 2012;36(3):477-490.

22. Saze Z, Schuler PJ, Hong CS, Cheng D, Jackson EK, Whiteside TL. Adenosine production by human B cells and B cell-mediated suppression of activated T cells. Blood. 2013;122(1):9-18.

23. Shen $P$, et al. IL-35-producing B cells are critical regulators of immunity during autoimmune and infectious diseases. Nature. 2014;507(7492):366-370.

24. Baba Y, Matsumoto M, Kurosaki T. Signals controlling the development and activity of regulatory B-lineage cells. Int Immunol. 2015;27(10):487-493

25. Knippenberg S, et al. Reduction in IL-10 producing B cells (Breg) in multiple sclerosis is accompanied by a reduced naïve/memory Breg ratio during a relapse but not in remission. J Neuroimmunol. 2011;239(1-2):80-86.

26. Hayashi M, et al. IL-10-producing regulatory $\mathrm{B}$ cells are decreased in patients with psoriasis.
J Dermatol Sci. 2016;81(2):93-100.

27. Fillatreau S, Sweenie CH, McGeachy MJ, Gray

$\mathrm{D}$, Anderton SM. B cells regulate autoimmunity by provision of IL-10. Nat Immunol. 2002;3(10):944-950.

28. Carter NA, Rosser EC, Mauri C. Interleukin-10 produced by B cells is crucial for the suppression of Th17/Th1 responses, induction of T regulatory type 1 cells and reduction of collagen-induced arthritis. Arthritis Res Ther. 2012;14(1):R32.

29. Mauri C, Gray D, Mushtaq N, Londei M. Prevention of arthritis by interleukin 10-producing $\mathrm{B}$ cells. J Exp Med. 2003;197(4):489-501.

30. Blair PA, et al. Selective targeting of B cells with agonistic anti-CD40 is an efficacious strategy for the generation of induced regulatory T2-like $\mathrm{B}$ cells and for the suppression of lupus in MRL/lpr mice. JImmunol. 2009;182(6):3492-3502.

31. Matsushita T, Yanaba K, Bouaziz JD, Fujimoto M, Tedder TF. Regulatory B cells inhibit EAE initiation in mice while other B cells promote disease progression. JClin Invest. 2008;118(10):3420-3430.

32. Rosser EC, et al. Regulatory B cells are induced by gut microbiota-driven interleukin- $1 \beta$ and interleukin-6 production. Nat Med. 2014;20(11):1334-1339.

33. Yoshizaki A, et al. Regulatory B cells control T-cell autoimmunity through IL-21dependent cognate interactions. Nature. 2012;491(7423):264-268.

34. Correale J, Farez M, Razzitte G. Helminth infections associated with multiple sclerosis induce regulatory B cells. Ann Neurol. 2008;64(2):187-199.

35. Schubert RD, et al. IFN- $\beta$ treatment requires $B$ cells for efficacy in neuroautoimmunity. J Immunol. 2015;194(5):2110-2116.

36. Grützke B, et al. Fingolimod treatment promotes regulatory phenotype and function of B cells. Ann Clin Transl Neurol. 2015;2(2):119-130.

37. Thompson SA, Jones JL, Cox AL, Compston DA, Coles AJ. B-cell reconstitution and BAFF after alemtuzumab (Campath-1H) treatment of multiple sclerosis. J Clin Immunol. 2010;30(1):99-105.

38. Heidt S, Hester J, Shankar S, Friend PJ, Wood KJ. $B$ cell repopulation after alemtuzumab induction-transient increase in transitional B cells and long-term dominance of naïve B cells. Am J Transplant. 2012;12(7):1784-1792.

39. Barr TA, et al. B cell depletion therapy ameliorates 
autoimmune disease through ablation of IL-6-producing B cells. JExp Med.2012;209(5):1001-1010.

40. Heinemann K, et al. Decreased IL-10(+) regulatory B cells (Bregs) in lupus nephritis patients. Scand J Rheumatol. 2016;45(4):312-316.

41. Gao N, et al. Impaired suppressive capacity of activation-induced regulatory B cells in systemic lupus erythematosus. Arthritis Rheumatol. 2014;66(10):2849-2861.

42. Ivashkiv LB, Donlin LT. Regulation of type I interferon responses. Immunology. 2014;14(1):36-49.

43. Ramos-Casals M, Soto MJ, Cuadrado MJ, Khamashta MA. Rituximab in systemic lupus erythematosus: A systematic review of off-label use in 188 cases. Lupus. 2009;18(9):767-776.

44. Aguiar R, Araujo C, Martins-Coelho G, Isenberg D. Use of rituximab in systemic lupus erythematosus: a single center experience over 14 years [published online ahead of print April 25, 2016]. Arthritis Care Res (Hoboken). https://doi.org/10.1002/acr.22921.

45. Anolik JH, et al. Delayed memory B cell recovery in peripheral blood and lymphoid tissue in systemic lupus erythematosus after B cell depletion therapy. Arthritis Rheum. 2007;56(9):3044-3056.

46. Palanichamy A, et al. Novel human transitional $B$ cell populations revealed by $B$ cell depletion therapy. J Immunol. 2009;182(10):5982-5993.

47. Wermeling F, Lind SM, Jordö ED, Cardell SL, Karlsson MC. Invariant NKT cells limit activation of autoreactive CD1d-positive B cells. J Exp Med. 2010;207(5):943-952.

48. Daien CI, et al. Regulatory B10 cells are decreased in patients with rheumatoid arthritis and are inversely correlated with disease activity. Arthritis Rheum. 2014;66(8):2037-2046.

49. Ma L, Liu B, Jiang Z, Jiang Y. Reduced numbers of regulatory B cells are negatively correlated with disease activity in patients with new-onset rheumatoid arthritis. Clin Rheumatol. 2014;33(2):187-195.

50. Cui D, et al. Changes in regulatory B cells and their relationship with rheumatoid arthritis disease activity. Clin Exp Med. 2015;15(3):285-292.

51. Kim J, Lee HJ, Yoo IS, Kang SW, Lee JH. Regulatory B cells are inversely associated with disease activity in rheumatoid arthritis. Yonsei Med J. 2014;55(5):1354-1358.

52. Amagai M. Pemphigus: autoimmunity to epidermal cell adhesion molecules. Adv Dermatol. 1996;11:319-352; discussion 353.

53. Colliou N, et al. Long-term remissions of severe pemphigus after rituximab therapy are associated with prolonged failure of desmoglein $B$ cell response. Sci Transl Med.2013;5(175):175ra30.

54. Kaser A, Zeissig S, Blumberg RS. Inflammatory bowel disease. Annu Rev Immunol. 2010;28:573-621.

55. Kleffel S, et al. Interleukin-10+ ${ }^{+}$regulatory B cells arise within antigen-experienced $\mathrm{CD} 4 \mathrm{O}^{+} \mathrm{B}$ cells to maintain tolerance to islet autoantigens. Dia- betes. 2015;64(1):158-171.

56. Mavropoulos A, et al. Breg cells are numerically decreased and functionally impaired in patients with systemic sclerosis. Arthritis Rheum. 2016;68(2):494-504.

57. Matsushita T, Hamaguchi Y, Hasegawa M, Takehara K, Fujimoto M. Decreased levels of regulatory B cells in patients with systemic sclerosis: association with autoantibody production and disease activity. Rheumatology (Oxford). 2016;55(2):263-267.

58. Shen P, Fillatreau S. Suppressive functions of $\mathrm{B}$ cells in infectious diseases. Int Immunol. 2015;27(10):513-519.

59. Siewe B, et al. Regulatory B cell frequency correlates with markers of HIV disease progression and attenuates anti-HIV CD $8{ }^{+} \mathrm{T}$ cell function in vitro. J Leukoc Biol. 2013;93(5):811-818.

60. Siewe B, et al. Regulatory B cells inhibit cytotoxic Tlymphocyte (CTL) activity and elimination of infected CD4 $\mathrm{T}$ cells after in vitro reactivation of HIV latent reservoirs. PLoS One. 2014;9(4):e92934.

61. Liu Y, et al. IL-10-producing regulatory B-cells suppressed effector T-cells but enhanced regulatory T-cells in chronic HBV infection. Clin Sci. 2016;130(11):907-919.

62. Lee JH, et al. Allergen-specific B cell subset responses in cow's milk allergy of late eczematous reactions in atopic dermatitis. Cell Immunol. 2010;262(1):44-51.

63. Noh J, et al. Characterisation of allergen-specific responses of IL-10-producing regulatory B cells (Br1) in Cow Milk Allergy. Cell Immunol. 2010;264(2):143-149.

64. Olkhanud PB, et al. Tumor-evoked regulatory B cells promote breast cancer metastasis by converting resting $\mathrm{CD}^{+} \mathrm{T}$ cells to $\mathrm{T}$-regulatory cells. Cancer Res. 2011;71(10):3505-3515.

65. Zhang Y, Gallastegui N, Rosenblatt JD. Regulatory B cells in anti-tumor immunity. Int Immunol. 2015;27(10):521-530.

66. Wejksza K, et al. Cancer-produced metabolites of 5-lipoxygenase induce tumor-evoked regulatory $B$ cells via peroxisome proliferator-activated receptor $\alpha$. J Immunol. 2013;190(6):2575-2584.

67. Han S, et al. Glioma cell-derived placental growth factor induces regulatory B cells. Int J Biochem Cell Biol. 2014;57:63-68

68. Horikawa M, Minard-Colin V, Matsushita T, Tedder TF. Regulatory B cell production of IL-10 inhibits lymphoma depletion during CD20 immunotherapy in mice. JClin Invest. 2011;121(11):4268-4280.

69. Nouël A, Simon Q, Jamin C, Pers JO, Hillion S. Regulatory B cells: an exciting target for future therapeutics in transplantation. Front Immunol. 2014;5:11.

70. Newell KA, et al. Identification of a B cell signature associated with renal transplant tolerance in humans. JClin Invest. 2010;120(6):1836-1847.

71. Cherukuri A, et al. Immunologic human renal allograft injury associates with an altered IL-10/
TNF- $\alpha$ expression ratio in regulatory B cells. J Am Soc Nephrol. 2014;25(7):1575-1585.

72. Shabir S, et al. Transitional B lymphocytes are associated with protection from kidney allograft rejection: a prospective study. Am J Transplant. 2015;15(5):1384-1391.

73. Sarvaria A, et al. $\mathrm{IL}-10^{+}$regulatory B cells are enriched in cord blood and may play a role in protection against GVHD after cord blood transplantation. Blood. 2016;128(10):1346-1361.

74. Okur FV, Brenner MK. Cellular immunotherapy of cancer. Methods Mol Biol. 2010;651:319-345.

75. Miyagaki T, Fujimoto M, Sato S. Regulatory B cells in human inflammatory and autoimmune diseases: from mouse models to clinical research. Int Immunol. 2015;27(10):495-504.

76. Mizoguchi A, Mizoguchi E, Takedatsu H, Blumberg RS, Bhan AK. Chronic intestinal inflammatory condition generates IL-10-producing regulatory B cell subset characterized by CD1d upregulation. Immunity. 2002;16(2):219-230.

77. Yamamoto R, et al. PD-1-PD-1 ligand interaction contributes to immunosuppressive microenvironment of Hodgkin lymphoma. Blood. 2008;111(6):3220-3224.

78. Ansell SM, et al. PD-1 blockade with nivolumab in relapsed or refractory Hodgkin's lymphoma. NEngl J Med. 2015;372(4):311-319.

79. Armand P, et al. Disabling immune tolerance by programmed death-1 blockade with pidilizumab after autologous hematopoietic stem-cell transplantation for diffuse large B-cell lymphoma: results of an international phase II trial. J Clin Oncol. 2013;31(33):4199-4206.

80. Rafei M, et al. A granulocyte-macrophage colony-stimulating factor and interleukin-15 fusokine induces a regulatory $B$ cell population with immune suppressive properties. Nat Med. 2009;15(9):1038-1045.

81. Yang $\mathrm{M}$, et al. Novel function of $\mathrm{B}$ cell-activating factor in the induction of IL-10-producing regulatory B cells. JImmunol. 2010;184(7):3321-3325.

82. Genestier L, Taillardet M, Mondiere P, Gheit H, Bella C, Defrance T. TLR agonists selectively promote terminal plasma cell differentiation of $B$ cell subsets specialized in thymus-independent responses. J Immunol. 2007;178(12):7779-7786.

83. Grammer AC, Lipsky PE. CD154-CD40 interactions mediate differentiation to plasma cells in healthy individuals and persons with systemic lupus erythematosus. Arthritis Rheum. 2002;46(6):1417-1429.

84. Wang RX, et al. Interleukin-35 induces regulatory $\mathrm{B}$ cells that suppress autoimmune disease. $\mathrm{Nat}$ Med.2014;20(6):633-641.

85. Maseda D, et al. Regulatory B10 cells differentiate into antibody-secreting cells after transient IL-10 production in vivo. J Immunol. 2012;188(3):1036-1048. 